und der zunehmenden Privatisierung öffentlicher Daseinsvorsorge, zum anderen in der Ausrichtung der Politik an den (Profit-)Interessen der Wirtschaft, bzw. der Großunternehmen (die in einem Gegensatz zu den Interessen der Bürger*innen und zur Demokratie gestellt werden). Der gesellschaftliche Ist-Zustand ist daher negativ konnotiert, die glücksverheißende Dimension ist auf die Zukunft gerichtet. Der sozialökologische Umbau verweist auf eine zukünftige ,andere‘ Gesellschaftsordnung, auch wenn deren Gestaltung in vielen Teilen implizit bleibt. Stattdessen werden vielfach Forderungen, die auf eine verfahrenspolitische Ebene abzielen, artikuliert. Insgesamt bleiben die Konturen eines sozial-ökologischen Umbaus auch aufgrund der geringen diskursiven Verbreitung unscharf.

Die implizite Annahme globaler Ungleichverteilung, welche im Narrativ des ,sozial-ökologischen Umbaus` und teilweise auch dem der ,ökologischen Modernisierung` zu Tage tritt, ermöglicht es im Rahmen der schreckensverheißenden Dimension einen Gegenwartsbezug herzustellen und Konsequenzen des Klimawandels im globalen Süden sichtbar zu machen. Aber auch bezüglich dieser Dimension bleibt die diskursive Wirkung des Narrativs marginalisiert und wird vom hegemonialen Katastrophenszenario der ,Deindustrialisierung‘ überlagert.

Politische Praktiken, die sich im Kontext des Narrativs des ,sozial-ökologischen Umbaus' zeigen, sind in Anlehnung an Glynos/Howarth (2007) von ihrer Ausrichtung her als transformativ einzustufen, da die Hindernisse für die positive $\mathrm{Zu}$ kunftsperspektive ausschließlich system-intern - in dem Fall in der ökonomischen Struktur des Energiesektors - beschrieben werden. In der zeitlichen Perspektive der glücksverheißenden Dimension wird durch das Nennen verfahrenspolitischer Maßnahmen jedoch überwiegend ein Gegenwartsbezug hergestellt, wodurch im Rahmen des Narrativs auch als reformistisch ausgerichtet zu wertende politische Praktiken abgebildet werden. Deren Wirkung wird allerdings in der Intention als transformativ, auf ein nur ansatzweise skizziertes Zukunftsprojekt des sozialökologischen Umbaus ausgerichtet, dargestellt.

\title{
5.4 ZUSAMMENFÜHRUNG UND DISKUSSION: POST-POLITIK DER „ENERGIEWENDE“
}

In Kapitel 5.1.2 (Kollokationsanalyse) habe ich die regelmäßigen Differenzbeziehungen von „Energiewende“, herausgearbeitet und in Kapitel 5.1.3 die Entwicklung dieser Muster im Kontext der Klassifizierung von Forderungen und somit von Hegemonie diskutiert und zusammengeführt. In Kapitel 5.2 und 5.3 habe ich durch Analyse von Story-Lines komplexitätsreduzierende Erzählungen von „Energiewende“ nachgezeichnet, die Bedeutungskämpfe analysiert und schließlich durch Benennen und Beschreiben der fantasmatischen Narrative den Rahmen des „Energiewende“ - Diskurses auf der Ebene der Fantasie sichtbar gemacht. Bereits in der computerbasierten Analyse, welche die oberste Diskursebene adressiert, deutet sich eine Tendenz zur Reduktion des politischen Diskurses über erneuerbare Energien auf technologisch und ökonomisch orientierte Themenfelder an. Diese Tendenz festigt sich in den fol- 
genden beiden Analyseschritten, die auf die mittlere Ebene der Artikulationsmuster (Story-Lines) sowie auf die unterste Diskursebene der zugrunde liegenden Narrative abzielen. Demnach werden insbesondere verfahrenspolitische, technokratische und managementorientierte politische Praktiken mit „Energiewende“ in Verbindung gebracht. Erik Swyngedouw $(2015,2010,2011)$ zeigt, inwiefern der Klimawandel und die mit ihm zusammenhängenden Politikbereiche anfällig für post-politische Repräsentationen sind (vgl. auch Kenis/Mathijs 2014: 148). Dies werde ich nun in einer Diskussion anhand einiger Beispiele, die nach ihrem chronologischen Auftreten im Diskursverlauf geordnet werden, am „Energiewende“-Diskurs vertiefen, dadurch die Ergebnisse von Kapitel 5 zusammenführen und so die Relevanz post-politischer Repräsentationen für diese Ergebnisse herausarbeiten.

\section{Einführung von „Energiewende“ in den Diskurs als vor-politisches Element}

In der computerbasierten Analyse (Kapitel 5.1) wird deutlich, dass der Diskurs um „Energiewende“ in der 14. sowie 15. Legislaturperiode überwiegend von der rotgrünen Bundesregierung initiiert wird, aber schwach ausgeprägt ist und „Energiewende" im parlamentarischen Raum kaum mit Bedeutung aufgeladen wird. In Kapitel 5.1.3 habe ich gezeigt, dass „Energiewende“ vor „Fukushima“ kaum als Identifikationsprinzip kritischer Forderungen bedient wird - eine Politisierung von „Energiewende“ findet kaum statt, der diskursive Raum um „Energiewende“ bleibt unbestimmt. Von der rot-grünen Bundesregierung wird meist eine explizite Artikulation von „Energiewende“ vermieden, auch weil es innerhalb der Regierungskoalition differente Positionen gibt und eine grundsätzliche Wende in der Energiepolitik nicht als Konsens erscheint (vgl. Kapitel 5.2.1). Der Atomausstieg wird zwar von der rotgrünen Bundesregierung der 14. und 15. Legislaturperiode als „Einstieg in eine andere Energiepolitik“ interpretiert (vgl. z.B.: SPD 25.11.1999: 6742). Nur selten wird aber explizit gemacht, auf welche politische Praktiken eine ,andere Energiepolitik“ genau abzielt - ein Energiekonzept legt die rot-grüne Regierung nicht vor. Der Ausbau erneuerbarer Energien wird isoliert von „Energiewende“ als Moment im Diskurs fixiert, wobei die Diskussion darüber überwiegend ohne Einbettung in ein energiepolitisches Gesamtkonzept erfolgt. Aufgrund und im Sinne dieser mangelnden Bedeutungsgenerierung und somit nicht stattfindenden Politisierung von „Energiewende“ in der 14. und 15. Legislaturperiode spreche ich hier von einer vor-politischen „Energiewende“. Die „Energiewende“ hat den parlamentarischen Raum noch nicht oder noch kaum erreicht. ${ }^{87}$

Gleichzeitig dominieren in den mit „Energiewende“ verbundenen Themenbereichen wie erneuerbare Energien oder Klimaschutz sowie dem fantasmatischen Narrativ ,ökologischer Modernisierung' bereits in der 14. und 15. Legislaturperiode postpolitische Artikulationen. Das heißt, dass der Diskurs um erneuerbare Energien größtenteils auf einer verfahrenspolitischen Ebene verbleibt ohne naturalisierte Strukturen in Frage zu stellen. Umweltpolitische Bezüge zeigen sich in der Kollokationsanalyse (vgl. Kapitel 5.1.2) erneuerbarer Energien überwiegend im Knotenpunkt „Klima-

87 Die Bezeichnung, vor-politisch' bezieht sich also rein auf den parlamentarischen Raum. Innerhalb sozialer Bewegungen würde die Analyse sicherlich anders ausfallen. 
schutz", während andere ökologische Aspekte nicht adressiert werden. Das fantasmatische Narrativ der ,ökologischen Modernisierung“ stellt die Energiepolitik nicht grundsätzlich in Frage, sondern zielt vor allem auf ein ökologisch regulierendes Eingreifen in die Marktwirtschaft ohne an grundsätzlichen Strukturen von Wirtschaftsund Gesellschaftsordnung anzusetzen, weswegen die politischen Praktiken, die sich aus dem Narrativ ableiten, als reformistisch klassifiziert wurden. Als glücksverheißende Zukunftsperspektive dient das Versprechen, die Stärkung der Volkswirtschaft mit einer ökologischeren Wirtschaftsweise zu verbinden. Dabei stehen marktwirtschaftlich-technische Bereiche im Vordergrund (vgl. Kapitel 5.3.1). Die Bedeutung der Technologie erneuerbarer Energien als Wirtschaftsfaktor für die gesamte Volkswirtschaft ist die argumentative Grundlage für die Rechtfertigung ihrer Förderung und daher eine wichtige Story-Line (vgl. Kapitel 5.2.3). Das Ziel der Förderung wird dabei ebenfalls von Anfang an marktorientiert eingebettet, das EEG als Marktanreizprogramm konstituiert (vgl. Kapitel 5.2.4). Insgesamt zeigt sich eine technokratisch-managementorientierte Ausrichtung, ökologischer Modernisierung ' unter dem Motto: „we have to change radically, but within the contours of the existing state of the situation" (Swyngedouw 2015: 4). Eine solche Politik ist von einem konsensualen Charakter geprägt, der sich auch in der breiten Zustimmung über alle Fraktionen zeigt, die in der 14. und 15. Legislaturperiode bezüglich der Förderung erneuerbarer Energien herrscht. Differenzen zeigen sich überwiegend in der verfahrenspolitischen Umsetzung der Förderung (vgl. z.B. CSU 25.02.2000: 8440; Kapitel 5.2.2) ${ }^{88}$

Der Klimawandel mit seinen Auswirkungen wird dabei zum Katastrophenszenario stilisiert, aus dem die Notwendigkeit einer ökologischen Modernisierung abgeleitet wird (vgl. Kapitel 5.3.1). Die Folgen des Klimawandels werden explizit allerdings nur an wenigen Stellen benannt, zum Beispiel in Form von Katastrophenszenarien (z.B. SPD 28.05.2004: 10247) (vgl. Kapitel 5.3.1). Hier wird eine apokalyptische Bedrohung skizziert, die die Dringlichkeit des Handelns in den Mittelpunkt stellt: „Wenn wir jetzt nicht handeln [...] geht uns [...] im wahrsten Sinne des Wortes die Luft aus“ (SPD 28.05.2003: 10234; vgl. Kapitel 5.3.1). Eine Zentralität von Angst kann allerdings zu einer Beschränkung des politischen Raums führen:

„The point is that when fear becomes the overwhelming sentiment, the space for asking fundamental political questions about our current society is strongly restrained. This situation can then be manipulated to impose an apparently broad consensus concerning a set of technocratic and market-driven measures“ (Kenis/Mathijs 2014: 152).

Dem schreckenserzeugenden Katastrophenszenario des Klimawandels wird andererseits keine positive Vision einer ,anderen Zukunft', einer , anderen Gesellschaft ${ }^{\star}$ entgegengestellt. Die glücksverheißende Dimension des fantasmatischen Narrativs ,ökologischer Modernisierung' zielt auf einen Erhalt der jetzigen Lebensumstände (vgl. Kapitel 5.3.1). Swyngedouw (2010: 219) spricht im Anschluss an Derrida (1992: 66)

88 Ein Dissens besteht zwar bezüglich des „Atomausstiegs“, den CDU, CSU und FDP als Gefahr für den „Standort Deutschland“ konstruieren (vgl. z.B.: CDU 14.12.2001: 20711; vgl. Kapitel 5.2.3), aber auch dieser wird - wie oben dargestellt von der rot-grünen Koalition nicht zwingend mit einem grundsätzlichen Wandel der Energiepolitik verbunden. 
von ,purer Negativität“. Gleichzeitig werden die Ursachen des Klimawandels in den Debatten kaum explizit genannt und nicht mit möglichen strukturellen Ursachen, bspw. innerhalb des ökonomischen Systems, kontextualisiert. So werden selbst dann, wenn von Klimaschutz oder Klimawandel die Rede ist, meist kaum politische Inhalte oder Strukturen adressiert.

Gleichzeitig scheint die Feststellung der Existenz des Problems ,Klimawandel Konsens zwischen allen im Zeitraum der Untersuchung im Bundestag vertretenen politischen Fraktionen zu sein. Die Bedeutung der Forderungen ,Klimaschutz' und ,erneuerbare Energien' bleibt zwischen den äquivalentiellen Fronten zwischen Befürwortung und Ablehnung einer „Energiewende“ somit unbestimmt (Laclau 2005: 132; vgl. Kapitel 5.1.3), sie sind nicht in eine starke kritische Äquivalenzkette eingebunden. Der Klimawandel erscheint so als Problem , an sich‘. Durch diese Externalisierung des Konfliktes um Klimaschutz auf ein „sozial entkörpertes [eig. Übersetzung]", da nicht im Kontext seiner sozial-ökologischen Verursachung thematisierten, $\mathrm{CO}_{2}$ erscheinen alle Akteur*innen in diesem Kampf vereint - es kommt zu einer „pseudo-Politisierung [eig. Übersetzung]“ (Kenis/Mathijs 2014: 151). Dies zeigt sich bspw. in der Diskussion um den Emissionshandel in der 15. Legislaturperiode, der prinzipiell breite Befürwortung findet und mit dem der Klimaschutz „effizient“ gemacht werden soll (GRÜNE 28.05.2004: 10237) ohne grundsätzliche ökonomische Strukturen zu verändern. Swyngedouw (2015: 5) argumentiert, dass es gerade die konsensuale Konstruktion von Klimaschutz sei, die für einen Entpolitisierungsprozess stehe. So werde einerseits das Ökologische, repräsentiert durch das Klima, politisch mobilisiert, während gleichzeitig die Sorge um das Klima die politische Dimension dadurch außer Kraft setze, dass die Mobilisierung ohne grundsätzliches - über eine verfahrenspolitische Ebene hinausgehendes - politisches Ziel erfolge (ebenda: 2). Die strukturellen, systembedingten Ursachen für den Klimawandel - geschweige denn anderer ökologischer Probleme - werden nicht thematisiert. Diese Tendenz der Entpolitisierung des Diskursgegenstandes lässt sich anhand der drei Analyseschritte für den „Energiewende“ - Diskurs nachzeichnen, wobei sie sich mit fortschreitendem Analyseprozess verstetigt. Nach Swyngedouw (2011: 258f.) bleibe die Natur somit letztlich ,extern'; das ,Andere' des Politischen - der politische Gehalt von ,Natur' werde geleugnet. Es handele sich um einen Vorgang der Entpolitisierung par excellance.

Stattdessen zeigt sich in der Analyse der Story-Lines eine Dominanz marktwirtschaftlicher, auf technischen Fortschritt sowie globalen Wettbewerb gerichteten Erzähl- und Deutungsmuster. So wird mit dem Emissionshandel, der Ökosteuer, dem EEG und anderen politischen Maßnahmen nach möglichst wirtschaftsfördernden (vgl. Kapitel 5.2.3), marktwirtschaftlich orientierten (vgl. Kapitel 5.2.4) oder ,preisgünstigsten“ (SPD 28.05.2004: 10247) Lösungen gesucht. Während eine Politik des Politischen den Klimawandel als Krisensymptom des ,Normalen“ sehen und die diesbezügliche Differenz in den politischen Fantasien herausarbeiten würde, bestehe der hegemoniale politische Diskurs auf eine Interpretation von Klimawandel als Anomalie des Systems, der durch ,eine Mobilisierung der inneren Dynamiken und Logiken des System, welches das Problem ursprünglich hervorgerufen habe [eig. Übersetzung]“, vorgebeugt werden solle (Swyngedouw 2011: 268f.). So werden die sozial-ökologischen Verhältnisse und Prozesse, durch die der Klimawandel verursacht werde, radikal verleugnet (Swyngedouw 2010: 222f.). 
Ein weiterer entpolitisierender Faktor des aufkommenden „Energiewende“Diskurses folgt aus seiner globalen Einordnung (vgl. Kapitel 5.2.6). So scheint zwischen der 14. und 16. Legislaturperiode „Energiewende“ vor allem dann relevant zu werden, wenn ein internationaler Kontext im Fokus steht, bspw. im Vorfeld der Renewables2004 Konferenz ${ }^{89}$ (vgl. Kapitel 5.1.2). Dabei wird die politische Verantwortung globalisiert (vgl. z.B. SPD 28.05.2004: 10234). Eine „Energiewende“ erscheint so als Projekt, das vor allem global eingeführt werden muss - und zwar am besten mit deutscher Technologie und deutschem Wissen (vgl. Kapitel 5.2.6). Die Notwendigkeit einer globalen „Energiewende“ wird aus dem Klimawandel sowie dem wachsenden Ressourcenverbrauch als universale humanitäre Bedrohung abgeleitet. ,Die Menschen' werden dabei nicht als heterogene Subjekte konstituiert, sondern als universale Opfer (Swyngedouw 2010: 221): „denn wegen der Emissionen und des Wettlaufs um knappe fossile Ressourcen gibt es für alle Menschen auf diesem Globus nur eine gemeinsame Zukunft“ (SPD 28.05.2004: 10234). Dabei werden konstitutive soziale Differenzen verschwiegen. Das universale politische Subjekt ,Mensch“ wird im Kontext eines antagonistischen Kampfes Klima vs. Mensch (Swyngedouw 2010: 221) bestimmt - alternative mögliche sozial-ökologische Konstellationen bleiben so außerhalb der Betrachtung, da jede andere antagonistische Spaltung verdeckt wird. Es scheinen zwar nicht alle Menschen im gleichen Ausmaß vom Klimawandel betroffen und für ihn verantwortlich, doch bleibt die Menschheit letztlich in ihrer grundsätzlichen Betroffenheit und Verantwortung vereint:

„Bisher hat ein Drittel der Welt, 2 Milliarden Menschen, keinen Zugang zu moderner Energie. [...]. Dabei ist aber eines klar: Entwicklungsländer dürfen und wollen nicht die Fehler wiederholen, die die Industrieländer bisher bei ihrer Energieversorgung gemacht haben, denn das hält unser Globus nicht aus“ (SPD 28.05.2004: 10234).

Letztlich wird durch die Gegenüberstellung Klima vs. Mensch die Mensch/Natur Dichotomie reproduziert (Swyngedouw 2011: 268f.; Smith 2008: 245) und vorherrschende gesellschaftliche Naturverhältnisse perpetuiert, was in Kapitel 6 in Bezug auf den Gesamtdiskurs noch genauer betrachtet wird. Globale Machtverhältnisse werden - hier zwischen ,Entwicklungsländern` und Industrieländern - naturalisiert und somit verdeckt. Das Verdecken des politischen Gehalts dieser Verhältnisse trägt zur Entpolitisierung des gesamten Diskurses bei. Auch dies wird in Kapitel 6.4 noch genauer betrachtet.

\section{Vermischung der fantasmatischen Narrative, ökologischer Modernisierung“ und ,marktwirtschaftlicher Energiepolitik}

Nachdem „Energiewende“ unter der rot-grünen Regierungskoalition also einerseits kaum politisiert wurde und andererseits gleichzeitig in den mit ihr in Verbindung gesetzten Themenbereichen und Forderungen post-politische Vorstellungen dominieren, verschwindet sie in der 16. Legislaturperiode fast gänzlich aus den bundespolitischen Debatten. Die große Koalition knüpft aber vielfach an die Artikulationsmus-

89 Die Anzahl der Vorfälle von „Energiewende“ in dieser Debatte ist die höchste der gesamten 15. Legislaturperiode (vgl. Tabelle 4 in Kapitel 4.2.2). 
ter/Story-Lines und Forderungen des Narrativs der ,ökologischen Modernisierung، an. Allerdings spricht sie dabei nicht explizit von ökologischer Modernisierung, sondern setzt die erneuerbaren Energien in den Kontext eines ,breiten Energiemixes“ und verwendet dabei den Begriff der ,Nachhaltigkeit' (vgl. Kapitel 5.2.1). So wird das zentrale Versprechen des fantasmatischen Narrativs ,ökologischer Modernisierung ' - die Vereinbarkeit von (markt)wirtschaftlichen und ökologischen Zielen -von der CDU/CSU durch Einbindung in die eigenen Äquivalenzketten angeeignet. Erneuerbare Energien sollen zum Bestandteil ,Sozialer Marktwirtschaft' werden (CDU 02.07.2009: 25872).$^{90}$ Spätestens in der 16. Legislaturperiode wird deutlich, dass sich die fantasmatischen Narrative ,ökologische Modernisierung' und ,marktwirtschaftliche Energiepolitik' hinsichtlich ihrer verfahrenspolitischen, technokratisch-managementorientierten Ausrichtung politischer Praktiken aneinander angleichen. Sie unterscheiden sich überwiegend im Stellenwert, der umweltpolitischen Themen innerhalb der auf Verfahrenspolitik beschränkten Dimensionen des Glücks zugewiesen wird, und in ihren Katastrophenszenarien (Folgen des Klimawandels vs. Deindustrialisierung) (vgl. Kapitel 5.3). Aufgrund der abnehmenden Artikulation ,ökologischer Modernisierung' (vgl. Abbildung 13) sowie der Aneignung von Artikulationsmustern durch das marktwirtschaftliche Narrativ, vermischen sich die fantasmatischen Narrative zunehmend.

In der 17. Legislaturperiode greift dann auch die FDP den Kern des Versprechens ,ökologischer Modernisierung ‘ - die Vereinbarkeit von ökologischen und (markt)wirtschaftlichen Zielen - auf. Dabei werden von der schwarz-gelben Koalition auch die wirtschaftlichen Gefahren, die vom Klimawandel ausgehen, hervorgehoben, während ökologische und soziale Krisenszenarien weiterhin ausgeblendet werden. Klimaschutz sei notwendig, um den Industriestandort nicht in seiner Wettbewerbsfähigkeit zu gefährden (17/3049: 2; vgl. Kapitel 5.3.2). Zur Rechtfertigung der Laufzeitverlängerung bindet die schwarz-gelbe Regierungskoalition Forderungen, die mit „Energiewende“ (z.B. „Umbau unseres Energieversorgungssystems“ (CDU 28.10. 2010: 7168)) und ,ökologischer Modernisierung“ (,das größte Modernisierungsprogramm für eine gesamte Volkswirtschaft“ (FDP 28.10.2010: 7201)) in Verbindung stehen in ihre Äquivalenzketten ein. Mit der Kernenergie als ,Brückentechnologie“ wird der Anschein erweckt, dass Klima und Wirtschaft auf einmal gerettet werden können (vgl. auch Swyngedouw 2011: 270f.). Die schwarz-gelbe Koalition durchbricht dabei die antagonistische Grenze zwischen Befürwortung und Gegnerschaft

90 Dennoch kommt es im Vorfeld der Bundestagswahl 2009 zumindest kurzzeitig zu einer Repolitisierung des Diskurses um „Atomausstieg“ und damit verbunden der Energiepolitik. Mit der CDU und der CSU sind zwei Parteien an der Regierung, die weiterhin offiziell erklären, den „Atomausstieg“ rückgängig machen zu wollen. GRÜNE und LINKE und im Vorfeld der Wahl auch die SPD konstituieren darüber hinaus zunehmend die, großen vier Energiekonzerne“ als Gefahr für den „Atomausstieg“ sowie für eine nachhaltige Energiepolitik. CDU/CSU und FDP werden dabei in ihrer Politik mit den Interessen dieser Konzerne äquivalenziert (vgl. z.B. SPD 02.07.2009: 25880). Dabei wird das Bild eines vermeintlichen Konsenses über den Ausbau der erneuerbaren Energien, das noch zu Beginn der großen Koalition der 16. Legislaturperiode auch von der SPD bedient wurde, durchbrochen (vgl. Kapitel 5.2.10). 
einer „Energiewende“ gezielt (vgl. Kapitel 5.1.3, Abbildung 11) und verschiebt diskursive Elemente über diese Grenze hinweg (vgl. auch Nonhoff 2006: 234).

In der Äquivalenzierung von ,,ideologiefrei“, „,marktorientiert“ und „technologieoffen“ (17/3050: 8) zeigt sich die entpolitisierende Wirkung des fantasmatischen Narrativs ,marktwirtschaftlicher Energiepolitik' deutlich (vgl. Kapitel 5.3.2). Diese wird durch die Konstruktion eines vermeintlichen (nationalen) Konsenses verstärkt: Die Energiepolitik als „Lebensader unserer Gesellschaft“ sollte „kein Streitthema“ sein, sondern ein gemeinsames Thema, ,weil wir dem Land zu dienen haben“ (CDU 28.10.2010: 7178). Eine Politisierung der Debatte durch die Opposition wird so als ,ideologisch “ und interessengeleitet delegitimiert, während die Prämissen der schwarz-gelben Politik naturalisiert werden. Letztlich wird eine Differenz zu den Zielsetzungen der Opposition bestritten: „Ich glaube sogar - weil wir so viel streiten und weil es hier in dieser Debatte so einen großen Streitgestus gibt -, dass das in Wahrheit ein Konsens in diesem Haus ist, dass wir das alle wollen" (CDU 28.10. 2010: 7178).

\section{Entpolitisierende Diskursmuster nach „Fukushima“}

„Fukushima“ ist eine Dislokation für diesen Diskurs, da es die Verknüpfung von ,technologieoffen', ,ideologiefrei' und ,Vernunft' unterminiert. Durch Reartikulation, d.h. durch die Herstellung neuer diskursiver Verknüpfungen, gelingt aber die Einbindung von „Energiewende“ in die hegemoniale Formation, wie in Kapitel 5.1.3 gezeigt wurde. Dabei lassen sich im Laufe der Analyseschritte im Wesentlichen drei Deutungsmuster ausmachen, die darauf zielen, das Postulat der Ideologiefreiheit für die eigene Politik nach „Fukushima“ zu erhalten, bzw. wiederherzustellen.

Erstens: „Fukushima“ wird als die erste nukleare Katastrophe konstituiert, die auf unerwartbares technisches Versagen (nicht auf menschliches) zurückzuführen (z.B. FDP 09.06.2011: 12967) und wissenschaftlich nicht vorhersehbar gewesen sei (Angela Merkel 17.03.2011: 10884). Die Ursache für die Dislokation wird so in einem ,Außen` gesucht, die Laufzeitverlängerung ihrem politischen Zusammenhang entzogen: Die Ursache von „Fukushima“ liege in einem technischen Versagen, das unabhängig von den politischen Akteur*innen zu bewerten sei und nun zu einer ,neuen Lage' führe: „Wenn das so ist, wenn also in einem so hoch entwickelten Land wie Japan das scheinbar Unmögliche möglich, das absolut Unwahrscheinliche Realität wurde, dann verändert das die Lage“ (Angela Merkel 17.03.2011: 10884f.). Um dieser ,neuen Lage' gerecht zu werden, wird in der Folge Expert*innen für energiepolitische Entscheidungen eine herausragende Rolle zugewiesen (vgl. Kapitel 5.2.9). Die Möglichkeit eines technischen Versagens in einem hochindustrialisierten Land erfordere eine Neubewertung unter wissenschaftlichen Gesichtspunkten. Diese Neubewertung erfolgt zunächst durch die ,Ethikkommission Sichere Energieversorgung ${ }^{6}$. Es erfolgt also eine Verschiebung aus dem Bereich des Politischen in die Wissenschaft. So erscheint die Entscheidung zum Atomausstieg in Verbindung mit der „Energiewende" als wissenschaftliche und weniger als politische Frage. Wissenschaftliche Expertise wird zur Garantie und Grundlage für die ,richtige“ politische Entscheidung erhoben (Swyngedouw 2010: 217). Die wissenschaftliche Neubewertung führt im nächsten Schritt zur Stilisierung der „Energiewende“ als ,neuer Konsens“.

Zweitens: Die „Energiewende“ wird als nationaler Konsens konstruiert: „Insofern ist es richtig, gemeinsam in einem gesellschaftlichen Konsens, mit der Ethik-Kom- 
mission und allen beteiligten gesellschaftlichen Gruppen, den Beschluss zu fassen, nach 2022 auf die Kernenergie zu verzichten“ (FDP 09.06.2011: 12967). Als handelndes Subjekt wird eine konstruierte nationale Gemeinschaft eingesetzt („Die Deutschen“ (CDU 30.06.2011: 13371); „Wir alle“ (Angela Merkel 09.06.2011: 12963)). Nach „Fukushima“ trägt der nationale Mythos, der nun mit „Energiewende“ verbunden wird (Angela Merkel 09.06.2011: 12963f.) dazu bei, Gegner*innen der „Energiewende“ zu integrieren - die identitätsstiftende Funktion der „Energiewende“ für „Deutschland“ gewinnt nun an Bedeutung (vgl. Kapitel 5.1.3). Gerade dadurch, dass die Knotenpunkte „Deutschland“ und „Energiewende“ offen sind für verschiedene Projektionen der politischen Fraktionen und ihrer jeweiligen Positionen, entsteht eine konsenserzeugende, entpolitisierende Wirkung (vgl. Kapitel 5.1.3; 5.2.6). Es wird eine gesellschaftliche Einigkeit konstruiert, bestehende Konflikte werden verdeckt und so eine Schließung des Diskurses verheißen (vgl. z.B. CDU 30.06. 2011: 13369). Hier wird also bereits kurz nach „Fukushima“ der Dissens über die grundsätzliche Gestaltung der „Energiewende“ aus dem vermeintlich konsensualen politischen Raum gedrängt. „Energiewende“-Politik wird auf eine verfahrenspolitische Ebene innerhalb gegebener Strukturen reduziert.

Drittens: Was innerhalb dieses, ,neuen Konsenses' als ,richtig' gilt, soll nicht durch ,Ideologie', sondern durch ,Vernunft' bestimmt werden. Während das Energiekonzept von schwarz-gelb ,vernünftig[...]“ sei, habe die rot-grüne Bundesregierung bei ihrem „Ausstiegsbeschluss einfach nur Ihre Ideologie befriedigt“ (FDP 09.06.2011: 12967). Wie zuvor die Kernenergie wird nun „Energiewende“ in einer Äquivalenzkette mit ,Vernunft', ,Marktorientierung' und ,Ideologiefreiheit' reartikuliert und so ihre marktorientierte Einbindung naturalisiert ${ }^{91}$ (vgl. Kapitel 5.2.4): „Das zeigt, wie weit Sie vom Markt, von der Realität und von ökonomischer Vernunft entfernt sind, meine Damen und Herren [...]. Wir treiben die Energiewende voran mit wirtschaftlicher Vernunft und sozialer Verantwortung“ (CDU 29.03.2012: 20307).

\section{Ausgrenzung des Ökologischen und des Sozialen aus dem diskursiven Raum nach „Fukushima“}

Die Probleme ,Klimawandel‘ und ,Atomausstieg‘ werden im Rahmen der „Energiewende“ zur ökonomischen Chance uminterpretiert, während Machtverhältnisse, z.B. zwischen Geschlechtern, Klassen und Weltregionen oder Produktionsverhältnisse aus dem politischen Raum gedrängt werden. Bereits in Kapitel 5.1.3 wurde gezeigt, dass

91 Politische Forderungen, die nicht mit einem solchen Verständnis der „Energiewende“ kompatibel sind, werden als, ideologisch', ,unwirtschaftlich“, ,emotional“ oder ,sozialistisch` abgespalten und abgewertet. Dies wird beispielsweise deutlich, als die LINKE 2012 fordert, die Befreiung der energieintensiven Industrie von der EEG-Umlage zu begrenzen: „Bei den ideologischen Ergüssen und sozialistischen Ausarbeitungen Ihrerseits fällt es manchmal schwer, gelassen zu bleiben, [...]. Meine Damen und Herren, ich weiß, dass Sie Probleme mit dem demokratischen Rechtsstaat und dem demokratischen System haben. Aber nicht Sie entscheiden über Recht und Unrecht; [...] Darüber entscheidet auch kein ZK oder Politbüro, sondern dieses Haus [...]. Meine Damen und Herren, wir haben es nicht nötig, uns mit solchen Leuten auf Anti-Marktwirtschafts-Debatten einzulassen“ (CDU 29.03.2012: 20292f). 
relativ schnell eine Begrenzung des diskursiven Raumes gelingt, was sich in der weiteren Analyse in der ökonomischen Ausrichtung der Story-Lines zeigt und durch Aussagen wie „Energiepolitik ist Wirtschaftspolitik“ (CSU 13.06.2013: 31584) reproduziert wird (vgl. Kapitel 5.2.3). Nonhoff (2007: 188) bezeichnet eine solche hegemoniale Strategie als „superdifferenzielle Grenzziehung“, die dazu führe, dass „,einzelne diskursive Elemente oder ganze Diskursregionen aus der Zugehörigkeit zu einem bestimmten diskursiven Raum verbannt [werden], indem ihre Differenz in gewisser Weise ,verstärkt" wird“. In diesem Fall werden bspw. „Sozial- und Arbeitsmarktpolitik“ (CSU 13.06.2013: 31584) explizit aus dem diskursiven Raum der Energiepolitik ausgeschlossen. Mit dieser Strategie wird die Ausbildung von „Energiewende“ als diskursivem Gegenstand grundlegend geprägt und die „Voraussetzung für die Möglichkeit von hegemonialer Praxis“ geschaffen (Nonhoff 2007: 188), die „Energiewende“ als ein (markt)wirtschaftspolitisches Feld konstituiert. ${ }^{92}$

Vor diesem Hintergrund erklärt sich auch, weshalb die vermeintlich hohen Kosten der „Energiewende“ eine zunehmend zentrale Rolle spielen, während sozialökologische Themenbereiche und Handlungsfelder im Hintergrund bleiben (vgl. Kapitel 5.2.5; vgl. auch Kapitel 5.1.2). Zudem erfolgt implizit eine diskursive Reduktion von „Energiewende“ auf den Strombereich. Darüber hinausgehende Bereiche wie die Wärmeversorgung oder der Verkehrsbereich werden vergleichsweise selten adressiert - eine energiepolitische Wende in diesen Bereichen scheint mit marktwirtschaftlichen Zielen schwieriger vereinbar zu sein. So erklärt die CDU mobilitätspolitische Ziele nicht gegen die Industrie durchsetzen zu wollen, denn es gehe ,nicht gegen den Markt, es geht nur mit dem Markt“" (CDU 13.06.2013: 31303).

Solche Grenzziehungen werden von den Oppositionsfraktionen nur unzureichend herausgefordert: Obwohl sich SPD und GRÜNE in einigen verfahrenspolitischen Positionen deutlich von der schwarz-gelben Regierungskoalition abgrenzen (z.B. Ausbauziele, Förderhöhe, Gestaltung der Einspeisevergütung), werden grundsätzliche marktwirtschaftliche Zielsetzungen von ihnen kaum in Frage gestellt und soziale sowie ökologische Themen in Verbindung mit „Energiewende“ zu selten gesetzt.

\section{Etablierung eines ,technokratisch-managementorientierten Ökokonsenses“}

In der 18. Legislaturperiode, in der die große Koalition das Ziel der Marktorientierung mit den Novellierungen des EEG offensiv weiter verfolgt, etabliert sich die marktwirtschaftliche Einbindung von „Energiewende“ weiter (vgl. Kapitel 5.2.4). Obwohl GRÜNE und LINKE die jeweilige Regierungspolitik kritisieren, bleibt die Artikulation einer ,anderen“ „Energiewende“ weitgehend aus. Nach „Fukushima“ werden grundlegende Glücks- und Katastrophenszenarien des fantasmatischen Narrativs ,ökologischer Modernisierung' auch von den GRÜNEN kaum noch bedient. Ihre Kritik an der Regierungspolitik beschränkt sich überwiegend auf die verfahrenspolitische Ebene. Die fantasmatischen Narrative ,ökologischer Modernisierung und ,marktwirtschaftlicher Energiepolitik` gehen damit in einem gemeinsamen ,technokratisch-managementorientierten Ökokonsens` auf, indem die Herausarbeitung von

92 Dies zeigt sich gegen Ende der 17. Legislaturperiode bspw. in der strikten Ablehnung der ,Rettung' der Solarindustrie (vgl. Kapitel 5.2.3). 
grundsätzlicher Differenz zwischen den Narrativen aufgegeben wird (vgl. Abbildung 14). ${ }^{93}$ Der ,technokratisch-managementorientierte Ökokonsens' kann nun als gemeinsames fantasmatisches Narrativ einer hegemonialen marktwirtschaftlichen Formation gewertet werden. ${ }^{94}$ Dieser ,Ökokonsens ${ }^{6}$ basiert zwar auf umweltpolitischen Forderungen, indem umweltpolitische Artikulationsmuster diskursiv angeeignet werden, gleichzeitig werden diese Forderungen aber permanent aus dem Bereich des Politischen in den Hintergrund gedrängt. Ihre Erfüllung wird der marktorientierten Verheißung des Erhalts von ,Wachstum und Wohlstand' untergeordnet.

Auch anhand der Debatte über den Ausbau der Netzinfrastruktur in der 17. und 18. Legislaturperiode lässt sich verdeutlichen, wie der Diskursrahmen durch den ,technokratisch-managementorientierten Ökokonsens“ determiniert wird. Die Netzinfrastruktur wird als „Dreh- und Angelpunkt“ (FDP 28.10.2010: 7201) der „Energiewende“ konstituiert. Die Planung der „Energiewende“ wird zum technischen Infrastrukturprojekt stilisiert. Die Debatte darüber orientiert sich zunehmend an großen Energie-Produktionszentren, bspw. im Rahmen von Offshore-Windenergie. Diskurse, die danach fragen, wie sozial-ökologische Anliegen mit technischen Herausforderungen integriert werden können, bleiben marginal (z.B. LINKE 01.06.2016: 16985f.; vgl. Kapitel 5.2.7).

Gleichzeitig wird der Netzausbau in der 18. Legislaturperiode zunehmend als Problem der „Energiewende“ konstituiert - er könne nicht mit dem Ausbau Schritt halten (vgl. Kapitel 5.2.7). Mit Artikulationen wie ,Überförderung` oder ,Ausbaukorridor' wird ein verlangsamter Umstieg auf erneuerbare Energien gefordert (vgl. Kapitel 5.2.2), die Artikulationen von „Energiewende“ durch SPD, CDU und CSU sind zunehmend von Ambivalenzen geprägt (vgl. Kapitel 5.3.2). So wird die „Energiewende" selbst Teil der schreckensverheißenden Dimension, indem bspw. der Eindruck erweckt wird, sie sei zu teuer, verursache eine „Kostenexplosion“ (z.B.: SPD 10.04.2014: 2433; Koalitionsvertrag 2013: 38) und schade der Wirtschaft (vgl. Kapitel 5.2.5; 5.3.2). Die SPD fordert vor dem Hintergrund des Katastrophenszenarios einer „Deindustrialisierung“ (SPD 27.06.2014: 3939f.) eine wirtschaftsorientierte „Energiewende“-Politik. Sie gibt in der 18. Legislaturperiode die Differenz zu wirt-

93 Dies bedeutet nicht, dass von den Akteur*innen, die innerhalb dieser Muster artikulieren, dieselben Interessen verfolgt werden. Es geht hier lediglich darum die diskursiven Muster in ihrer Wirkung zu betrachten und zu klassifizieren (vgl. dazu auch Kapitel 7.5). Es handelt sich also lediglich um einen scheinbaren Konsens, der durch eine Aneignung ökologisch orientierter Artikulationsmuster auf der einen Seite und eine fehlende Herausarbeitung der Differenz auf der anderen Seite erzeugt wird. Auch wenn es sich also nicht um einen ,tatsächlichen Konsens‘ im Sinne eines Konsenses über politische Ziele oder Interessen handelt, entsteht im Diskurs der Anschein und mindestens teilweise auch die Wirkung eines solchen.

94 In Kapitel 5.1.3 habe ich gezeigt, dass sich die hegemoniale Formation, welche ich über ihre Abgrenzung zur „Energiewende“ definiert habe, nach „Fukushima“ den Knotenpunkt „Energiewende“ artikulatorisch aneignet. Dadurch ergibt sich eine (auch bezüglich der in ihr wirkenden Akteur*innen) erweiterte hegemoniale Formation, die sich vor allem durch die regelmäßige marktwirtschaftliche Einbindung von „Energiewende“ auszeichnet und, die ich daher hier als hegemoniale marktwirtschaftliche Formation bezeichne. 
schaftsliberalen Positionen - und somit wie gezeigt auch das von ihr mitinitialisierte Narrativ einer, ökologischen Modernisierung - weitgehend auf und schließt sich dem Diskurs einer konsensualen, ,vernünftigen“ „Energiewende“ im Gegensatz zu „,ideologischen Kampfparolen“ an (SPD 01.06.2016 16979; vgl. Kapitel 5.2.3). Hier zeigt sich: Die diskursive marktwirtschaftliche Einbindung von „Energiewende“, die einerseits als Lösung präsentiert wird, bietet gleichzeitig die Grundlage für ihre Delegitimation. Anstatt sozial-ökologischer Krisen oder den Folgen des Klimawandels, bestimmen mit der auf diese Weise ,sozial-ökologisch entbetteten“ „Energiewende“ selbst in Verbindung gebrachte Risiken, bspw. in Form einer Bedrohung für die Wirtschaft, das Katastrophenszenario. ,Andere“ Perspektiven auf die „Energiewende“, werden als "sozialistische[...] Industriepolitik“ (CSU 13.06.2013: 31585), „wie in der DDR“ (CDU 24.03.2011: 11284) delegitimiert (vgl. Kapitel 5.3.2) und als extern verortete Gefahr für eine marktwirtschaftliche Energiepolitik herangezogen.

\section{Abbildung 14: Vermischung der fantasmatischen Narrative}

,ökologische Modernisierung ' und , marktwirtschaftliche Energiepolitik ‘ im ,technokratisch-managementorientierten Ökokonsens ‘

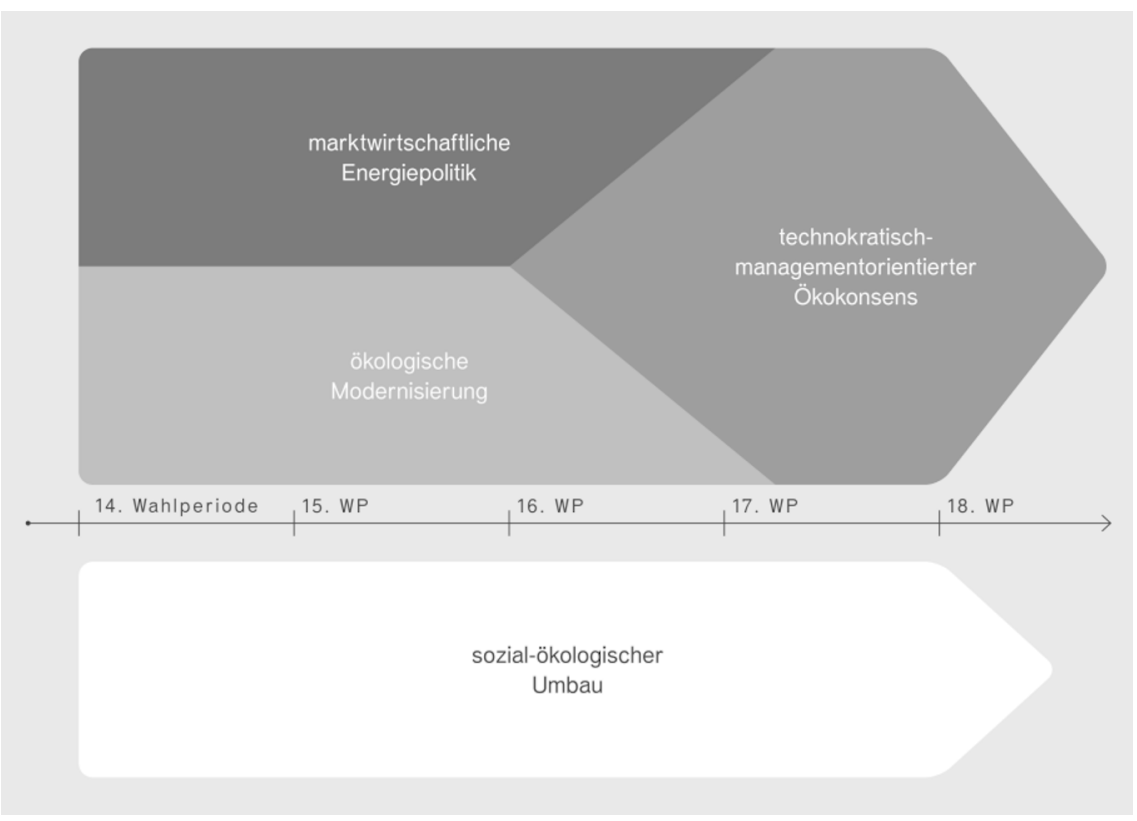

Quelle: eigene Darstellung

\section{Schlussfolgerungen}

Insgesamt zeigt sich in den Debatten zur „Energiewende“ nach „Fukushima“ ein Post-Politisierungsprozess, der sich mit Swyngedouw (2010: 216) als „Kolonisierung des Politischen durch die Verfahrensebene [eig. Übersetzung]“ bezeichnen lässt. Die politische Lenkung zentriert sich auf das technische und wirtschaftliche Management sowie die Verwaltung der „Energiewende“, wobei bestehende Verhältnisse im Bereich des Sozialen, der Produktion oder des Sozial-Ökologischen nicht angetastet werden (vgl. auch Swyngedouw 2015: 5). „This post-political frame is structured 
around the perceived inevitability of capitalism and a market economy as the basic organizational structure of the social and economic order, for which there is no alternative" (Swyngedouw 2010: 215). Wenn nach Glynos/Howarth (2007) die politische Realität der Fantasie bedarf, um sich zu konstituieren, und - wie nach „Fukushima“ eine große politische Mehrheit (aus Teilen von CDU, CSU, FDP, SPD und zum Teil auch GRÜNEN ${ }^{95}$ ) innerhalb eines fantasmatischen Narrativs artikuliert, welches die politische Realität aus einer spezifischen Perspektive heraus beschreibt - hier als technokratisch-managementorientierter Ökokonsens bezeichnet -, dann zeigt dies, dass die Naturalisierung dieser spezifischen politischen Realität gelungen ist, bei gleichzeitiger Verdeckung der Nicht-Naturgegebenheit sozialer Realität sowie der politischen Dimension ihrer Praktiken (vgl. Glynos/Howarth 2007: 145). Das verdeutlicht: Die entpolitisierende Wirkung des technokratisch-managementorientierten Ökokonsenses ist ein Symptom seiner Hegemonialität. Der Diskurs um „Energiewende" zielt demnach nicht (mehr) in erster Linie auf die Umsetzung umweltpolitischer Forderungen, sondern auf die Ausbreitung und den Erhalt der hegemonialen marktwirtschaftlichen Formation. „Energiewende“ entspricht nun in etwa dem, was Laclau (2007: 31) als „leeren Signifikanten“ bezeichnet - ein Signifikant, der kein Signifikat (mehr) hat. Die differentielle Identität von „Energiewende“ wurde von einer konsensorientierten Diskursformation aufgegeben, „um die rein äquivalentielle Identität eines gemeinschaftlichen Raumes als solches zu repräsentieren“ (Laclau 2002: 72). „Energiewende“ wurde nach „Fukushima“ zu einer umfassenden Forderung erhoben, mit dem Ziel den Bruch in den Artikulationen der hegemonialen Formation, die bis „Fukushima“ am alten Energiesystem festgehalten hatte, zu verdecken und wurde zur Einschreibungsfläche für überwiegend technokratisch-managementorientierte politische Praktiken, die letztlich mit einer Ausbreitung der hegemonialen Formation verbunden waren. Wie bei Laclau (2002: 74) ist zumindest für die Zeit nach „Fukushima“ die Präsenz des leeren Signifikanten „Energiewende“ „die eigentliche Bedingung für Hegemonie“.

Politische Entscheidungsfindung erscheint zunehmend als Frage von Expert*innenwissen und nicht als Ergebnis politischer Positionierung (Swyngedouw 2010: 225f.). Die Debatten drehen sich zum großen Teil um die Art der Technologie, den Zeitpunkt ihrer Einsetzung oder die Höhe ihrer Förderung. Die Natur wird dabei zwar auf unpolitische Weise in den Raum des Politischen eingeführt (ebenda: 216). Dies wird auch dadurch deutlich, dass gesellschaftliche Naturverhältnisse oder Annahmen über die Natur den Debatten über die „Energiewende“ permanent zu Grunde liegen, ja sogar ihr Ausgangspunkt sind, aber so gut wie nie explizit genannt werden - von Natur wird nicht gesprochen und wenn, dann findet eine Reduktion auf Klimaschutz statt (vgl. Kapitel 5.1.3). Die eigentliche politische Angelegenheit aber - die sozial-ökologische Krise - wird auf ein Terrain außerhalb der Debatte verlagert (vgl. Swyngedouw 2010: 217). Das eigentlich Politische wird aus dem Politischen extrahiert. Die Folgen des Klimawandels in ihrer schreckensverheißenden Dimension ver-

95 Wie bereits in Kapitel 5.1.3 verdeutlicht, sind die Fraktionsgrenzen nicht immer mit den Grenzen diskursiver Formationen identisch. So brechen bspw. die GRÜNEN und in einigen Fällen auch die SPD immer wieder aus dem technokratisch-managementorientierten Ökokonsens aus, indem sie sozial-ökologische Themen in den Blick holen. 
schwinden zunehmend aus den Debatten - möglicherweise, weil zentrale Herausforderungen des Klimaschutzes mit der beschlossenen „Energiewende“ zunächst als gelöst gelten, möglicherweise aber auch, weil ihre konkrete Benennung den Raum für die Herausarbeitung von Differenz zu weit öffnen würde und somit die auf Konsens ausgerichtete politische Praxis gefährden könnte. ${ }^{96}$ In Bezug auf die „Energiewende" hat die marktwirtschaftliche hegemoniale Formation umweltpolitische Forderungen nicht nur anerkannt, sondern konnte auch die Überzeugung etablieren, dass sie innerhalb der Marktwirtschaft bearbeitet werden können. Umweltpolitische Forderungen aus der Bevölkerung - z.B. aus der Anti-Atom-Bewegung - wurden so im Rahmen der marktwirtschaftlichen Hegemonie in den Diskurs aufgenommen und gleichzeitig auch für marktwirtschaftliche Interessen mobilisiert (vgl. auch Swyngedouw 2010: 223f.). Letztlich werden so sozial-ökologische Probleme nicht gelöst, sondern durch eine Relegitimation gegenwärtiger (westlicher) Lebensstile und vorherrschender politischer Interessen verstärkt (vgl. auch Brand u.a. 2009: 12) ${ }^{97}$ Im Zuge ihrer Integration in die hegemoniale marktwirtschaftliche Formation verbessert sich auch das „Image“ von Umweltschützer*innen (SPD 09.09.2004: 11256), die nun nicht mehr als politische Gegner*innen bekämpft werden. Sie seien nun nicht mehr die „Miesmacher, Müsliesser“ sondern „die Wegbereiter zu neuem Optimismus in Wirtschaft und Gesellschaft“ (SPD 09.09.2004: 11256).

Der Staat werde so zu einem Verfahrensakteur, der innerhalb eines neoliberalen Konsenses die Bedürfnisse des Marktes bediene und bei dem Regierungsführung auf die Verfahrens- und Verwaltungsebene reduziert werde, auf welcher der Staat vermeintlich gemeinsam mit Expert*innen agiere (Swyngedouw 2010: 227). Innerhalb dieser Sphäre des Nicht-Konfliktiven, innerhalb dieses technokratisch-managementorientierten Regelungssystems, kann quasi alles diskutiert werden, ist Dissens erlaubt, sind auch Positionswechsel möglich. ${ }^{98}$ Was diesen Rahmen aber überschreitet, erscheint „hoffnungslos aus der Zeit gefallen [eig. Übersetzung“ (vgl. z.B. den DDRVorwurf gegenüber der LINKEN) (Swyngedouw 2011: 267), unvernünftig und/oder unverantwortlich (vgl. Kapitel 5.3.2).$^{99}$ An einer inhaltlichen, d.h. über den Vorwurf der ,Rückständigkeit` hinausgehenden, Auseinandersetzung mit konkurrierenden Vi-

96 Die relative Häufigkeit von Klimawandel* im Gesamtkorpus ist für die 16. Legislaturperiode am höchsten.

97 Dass dieser Schein nicht dauerhaft aufrechterhalten werden kann, sondern durch die sozialökologische Krise, durch die „Spuren ihres Ausschlusses“ (vgl. Mouffe 2000: 147) permanent disloziert wird, zeigt sich in gegenwärtig erstarkenden Protesten gegen die Klimaschutz-Politik.

98 Deutlich wird dies auch in den wechselnden Positionierungen der großen Fraktionen, v.a. CDU/CSU und SPD.

99 Durch ihre Abweichung vom konsensualen, technokratisch-marktwirtschaftlichen Konsens erscheint die LINKE nicht als gleichwertige, respektable Diskurspartnerin, da ihre Positionen nicht als ,vernünftig' und somit nicht, verantwortungsvoll' gelten. Vor dem Hintergrund dieser Abwertung erscheint ein Ignorieren ihrer inhaltlichen Positionen legitim, eine politische Auseinandersetzung darüber geradezu niveaulos: „Meine Damen und Herren, wir haben es nicht nötig, uns mit solchen Leuten auf Anti-Marktwirtschafts-Debatten einzulassen“ (CDU 29.03.2012: 20292f). 
sionen sozial-ökologischer Zukünfte besteht im Rahmen der hegemonialen Formation kein Interesse, da die Alternativlosigkeit marktwirtschaftlicher Einbindung in Frage gestellt würde. So bleibt eine inhaltlich-politische Auseinandersetzung aus und die Fantasie eines sozial-ökologischen Umbaus unscharf. Die mit dieser Abweichung von der Norm verbundene soziale Ächtung verdeutlicht nicht nur, wie Ansichten, die den technokratisch-managementorientierten, bzw. marktwirtschaftlichen Konsens verlassen und alternative sozial-ökologische Arrangements in den Blick holen, zum Schweigen gebracht werden, es offenbart auch den beharrenden Kern dieses Konsenses (vgl. auch Swyngedouw 2010: 228). Denn vorherrschende soziale Verhältnisse, Produktionsverhältnisse und gesellschaftliche Naturverhältnisse sollen eben gerade nicht verändert werden.

Die Aufgabe einer pluralistischen demokratischen Politik bestehe nach Mouffe darin, die „Spuren der Macht und des Ausschlusses“ ins Zentrum zu rücken, sie sichtbar zu machen und somit in das Politische wiedereinzuführen (Mouffe 2000: 149). Dies sei ein unendlicher Prozess, denn der Antagonismus könne nicht ausgelöscht werden. Aber seinen verschiedensten Formen sollte in einem pluralistischen System Raum gegeben werden, sodass sich Identifikationsmöglichkeiten um ,wirkliche Alternativen' herum bieten und die gegenwärtige post-politische Vorstellung von Alternativlosigkeit gesprengt werden könne (Mouffe 2000: 149). Aus einem solchen Anspruch heraus seien demokratische Räume als Grundlage und Bedingung für ,andere' sozial-ökologische Arrangements zurückzuerobern, sozial-ökologische Zukünfte sollten positiv artikuliert und benannt werden sowie anerkannter Gegenstand von Konflikt werden (Swyngedouw 2011: 273). Das Politische am Umgang mit Natur, an den gesellschaftlichen Naturverhältnissen, an der Spaltung des Sozialen müsse sichtbar gemacht werden (ebenda). Die Ausschlüsse des hegemonialen Konsenses können so zum Ansatzpunkt von Repolitisierung werden. Solche Ansatzpunkte werden im folgenden Kapitel herausgearbeitet und mit dem Vorsorgenden Wirtschaften gleichzeitig in den Kontext von möglichen ,Alternativen“ im Hinblick auf Transformation gestellt. 
